\title{
Il button-hole: può migliorare la vita dell'emodializzato?
}

\author{
Francesco Bruni
}

U.O.C. di Nefrologia e Dialisi ASUR Marche, Area Vasta n 5, Ascoli Piceno-San Benedetto del Tronto (AP)

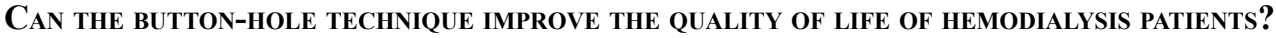

Abstract. For about 30 years the button-hole (B-H) technique has been used in several medical centers throughout the world, yielding contrasting results as for the survival of arteriovenous fistula, infections, hematomas, and pain during needle insertion. In this study we report the case of a medium-scale medical center that has been using the B-H technique for 4 years with satisfying results. These are likely to derive from the careful disinfection carried out, the supporting role of informatics tools and, particularly, from the strong determination of the caregivers, encouraged by the first positive results.
\end{abstract}

Key words: Vascular access, Button-hole, Quality of life

Conflict of interest: None.

Financial support: None.

Accettato: 6 Febbraio 2014

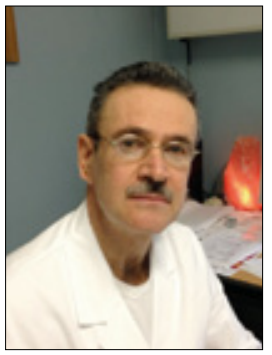

Francesco Bruni

\section{Introduzione}

Ho letto con interesse la revisione dei dati della letteratura sulla tecnica di punzione a sito costante (Button-Hole), pubblicata sul GTN\&D nell'ultimo numero del 2013, del Dr. Marcello Napoli (1), convinto (e con me è convinta l'intera comunità dei nefrologi) che la corretta gestione dell'accesso vascolare dei pazienti emodializzati viene a condizionare enormemente sia la qualità di vita dei nostri pazienti che la sopravvivenza stessa: il passaggio dalla fistola artero-venosa al catetere venoso centrale, come risulta dai dati della letteratura, purtroppo dimezza l'aspettativa di vita.

Qualsiasi intervento che possa migliorare la sopravvivenza dell'accesso vascolare con una FAV nativa deve essere visto come un tentativo di allungamento della vita e della sua qualità. Quanto riportato dal Dr. Marcello Napoli sulla tecnica B-H, che viene utilizzata sporadicamente in vari Centri da circa 30 anni (2), è esattamente quanto riportato in letteratura: ci sono studi che descrivono tale metodica come ottimale e studi che avanzano dubbi sui suoi reali vantaggi, anzi ne mettono in dubbio l'utilità e creano il sospetto che possa essere dannosa per il paziente, con un incremento di dolore locale, di infezioni, di ematomi e di sanguinamento.

Ho ritenuto, quindi, importante riportare l'esperienza della tecnica B-H, maturata in un Centro di dimensioni medio-piccole (78 pazienti in emodialisi), che utilizza tale tecnica nel $100 \%$ dei pazienti emodializzati dotati di FAV con vasi nativi (67 pazienti).

\section{Storia di una puntura}

Circa 4 anni fa, a seguito della presentazione della tecnica B-H da parte di una ditta che commercializza gli aghi smussi, i medici e gli infermieri del nostro Centro hanno avuto modo di approfondire la conoscenza di questa modalità di utilizzo dell'accesso vascolare.

Abbiamo fatto ricerche sull'argomento sulla letteratura mondiale, senza riuscire a trovare conclusioni convincenti e definitive sui possibili vantaggi.

Nello stesso periodo, il nostro Centro, essendo in una località turistica, ha ospitato per alcune sedute di emodialisi una paziente proveniente dall'Australia, che utilizzava la tecnica B-H da circa 4 anni. Siamo stati particolarmente colpiti dall'assenza di aneurismi, dalla facilità dell'auto-punzione, dall'assenza di infezioni e dall'apparente assenza di dolore durante la manovra di infissione degli aghi (la paziente aveva portato con sé gli aghi smussi).

La dimostrazione pratica della paziente australiana e l'autorevolezza della specialista che aveva fatto la presentazione della metodica ci convinsero che la tecnica B-H potesse essere una valida alternativa alla punzione tradizionale a scala di corda e ad area. Nell'Aprile del 2010, abbiamo deciso di iniziare questa esperienza, con tutti i timori del caso, che riguardavano soprattutto l'infezione, la complicanza più temibile, e la cura dell'inclinazione dell'ago, da mantenere costante anche nelle punzioni successive. Per quanto riguarda la prevenzione delle infezioni, abbiamo curato con estrema attenzione la rimozione completa della crosticina (3), cercando di ammorbidirla prima e di non traumatizzare la cute circostante. Abbiamo testato i disinfettanti più idonei, anche personalizzando la scelta in caso di intolleranza, cercando di ri- 
durre al minimo l'uso di quelli più irritanti.

Per mantenere l'inclinazione dell'ago costante per le sedute successive, almeno per le prime 3, l'operatore era sempre lo stesso, come sempre lo stesso era un secondo operatore che osservava la punzione e che sarebbe subentrato nelle punzioni successive. In sintesi, l'operatore che subentrava doveva assistere alle tre punzioni precedenti.

Per una maggiore sicurezza, prima di partire con la metodica, ci siamo dotati di una piccola telecamera per filmare la punzione e per creare un archivio sul computer del reparto, utile per gli operatori che sarebbero subentrati in seguito; una foto dell'inclinazione dell'ago veniva allegata alla cartellina di dialisi.

Dopo circa 8 punzioni, l'ago tradizionale (tagliente) veniva sostituito con l'ago smusso.

Gradualmente, il numero dei pazienti che utilizzavano la tecnica B-H è aumentato parallelamente al numero degli operatori che erano in grado di effettuarla.

A distanza di 1 anno, tutti i pazienti portatori di una FAV nativa utilizzavano la tecnica B-H e tutti gli infermieri erano in grado di utilizzare tale tecnica.

A distanza di circa 4 anni, possiamo sicuramente affermare che la punzione a sito costante della FAV ha avuto i seguenti risvolti positivi:

1) riduzione della formazione degli aneurismi

2) maggiore durata della FAV

3) dolore minore nel posizionamento degli aghi

4) netta diminuzione dello stress del paziente e dell'operatore dovuto alla ricerca del punto sempre nuovo di infissione degli aghi.

$\mathrm{Ci}$ sono stati anche aspetti che all'inizio potevano sembrare negativi, con il tempo, o sono stati superati o sono diventati delle opportunità.

- Le difficoltà organizzative per far coincidere i turni degli operatori con quelli dei pazienti che iniziavano la metodica, dopo il primo periodo, sono state ovviate grazie agli ausili informatici

- Il tempo di posizionamento degli aghi è aumentato per la necessità di rimuovere la crosticina, ma i pazienti sono diventati meno insofferenti durante le operazioni di inizio dialisi avendo preso maggiormente coscienza che "si lavora per loro".

- Al solo sospetto di infezione il punto di infissione deve essere cambiato, la scelta del nuovo punto va molto ponderata poiché potrebbe essere utilizzata per un tempo indefinito, ciò favorisce il dialogo fra gli operatori.

Tuttora, sia gli operatori che, soprattutto, i pazienti del nostro Centro non tornerebbero mai alla metodica tradizionale di punzione della FAV.

Queste mie osservazioni sono un sunto dell'esperienza del nostro Centro sulla metodica B-H; il mio ruolo come Direttore dell'U.O.C di Nefrologia e Dialisi è stato quello di stimolo, di incoraggiamento e di consulenza, soprattutto nei momenti di difficoltà.

Dal momento che ritengo che un grande merito per i risultati conseguiti vada riconosciuto al personale infermieristico, che ha saputo dedicare passione, dedizione e competenza crescenti, come da accordi con il Direttore della Rivista, nel prossimo numero saranno gli infermieri del Centro, che ho l'onore e l'onere di dirigere, a raccontare come hanno vissuto tale esperienza, con i dettagli organizzativi e metodologici, i risultati clinici e la relativa iconografia.

\section{Riassunto}

Da circa 30 anni, la tecnica di punzione a sito costante, Button-Hole $(\mathrm{B}-\mathrm{H})$, è utilizzata in vari Centri del mondo con risultati contrastanti per quanto riguarda la sopravvivenza della fistola artero-venosa (FAV), le infezioni, gli ematomi e il dolore nel posizionamento degli aghi. In questo lavoro, è riportata l'esperienza di un Centro di dimensioni mediopiccole che da circa 4 anni utilizza tale tecnica con risultati soddisfacenti; presumibilmente, tali risultati sono dovuti all'adozione di rigorosi protocolli di disinfezione, all'ausilio di strumenti informatici e, soprattutto, alla caparbietà degli operatori, incoraggiati dai primi risultati positivi.

Parole chiave: Accesso vascolare, Punzione a occhiello, Qualità di vita

Dichiarazione di conflitto di interessi: L'Autore dichiara di non avere conflitto di interesse.

Contributi economici agli Autori: L'Autore dichiara di non aver ricevuto sponsorizzazioni economiche per la preparazione dell'articolo.

Indirizzo dell'Autore:

Dr. Francesco Bruni

U.O.C. di Nefrologia e Dialisi

ASUR Marche, Area Vasta $n^{\circ} 5$

P.O. Madonna del Soccorso

63039 San Benedetto del Tronto (AP)

e

P.O.C. e G. Mazzoni

63100 Ascoli Piceno

Francesco.Bruni@sanita.marche.it

\section{Bibliografia}

1. Napoli M. Il Buttonhole: come trasformare una fistola in un catetere. Giornale di Tecniche Nefrologiche \& Dialitiche 2013; 25: 290-2.

2. Twardowski ZJ, Kubara H. Different sites versus constant sites of needle insertion into arteriovenous fistulas for treatment by repeated dialysis. Dial Transplant 1979; 8: 978-80.

3. Pegoraro M. La puntura a occhiello o buttonhole: quali sono le cose che "fanno la differenza". Una revisione della letteratura infermieristica. Giornale di Tecniche Nefrologiche \& Dialitiche 2013; 25 (2): 118-25. 